OPEN ACCESS

Edited by:

Martin G. Klotz,

Queens College (CUNY), USA

Reviewed by:

Praveen Rao Jurvadi,

Duke University, USA

Francine Govers,

Wageningen University and Research Centre, Netherlands

*Correspondence:

Ruey-Fen Liou

rfliou@ntu.edu.tw

tThese authors have contributed equally to this work.

Specialty section: This article was submitted to Fungi and Their Interactions,

a section of the journal

Frontiers in Microbiology

Received: 25 August 2016 Accepted: 27 March 2017 Published: 19 April 2017

Citation:

Hwu F-Y, Lai M-W and Liou R-F (2017) PpMID1 Plays a Role in the Asexual Development and Virulence of Phytophthora parasitica. Front. Microbiol. 8:610. doi: 10.3389/fmicb.2017.00610

\section{PpMID1 Plays a Role in the Asexual Development and Virulence of Phytophthora parasitica}

\author{
Fang-Yu Hwu', Ming-Wei Lait and Ruey-Fen Liou* \\ Department of Plant Pathology and Microbiology, National Taiwan University, Taipei, Taiwan
}

Phytophthora parasitica is a notorious oomycete pathogen that causes severe disease in a wide variety of crop species. Infection of plants involves mainly its asexual life stage, including papillate sporangia and biflagellated zoospores, which are the primary dispersal and infection agents of this pathogen. Calcium signaling has been thought as the key regulator for sporangium formation and zoospore differentiation. However, not much is known about the molecular players involved in these processes. In Saccharomyces cerevisiae, mating pheromone-induced death 1 (MID1) encodes a component of a putative calcium channel. Here, we identified and characterized the function of PpMID1, an MID1 homolog from P. parasitica. The expression of PpMID1 was high in sporangia. Gene silencing of PpMID1 resulted in the formation of sporangia that lacked papilla and showed a tendency for direct germination. Notably, in response to cold shock to induce zoospore formation, these sporangia showed no sign of cytoplasmic cleavage and thereby failed to form zoospores. Nonetheless, the addition of $\mathrm{CaCl}_{2}$ or $\mathrm{MgCl}_{2}$ partially recovered the silenced sporangia phenotype, with the formation of papillate sporangia similar to those of the wild type and the release of zoospores upon cold shock. As well, virulence toward Nicotiana benthamiana was reduced in the PpMID1-silenced transformants. These results indicate a role of PpMID1 in the asexual development and virulence of $P$. parasitica.

Keywords: asexual development, calcium signaling, MID1, Phytophthora, sporangia

\section{INTRODUCTION}

Phytophthora belongs to oomycetes that resemble true fungi in morphology and pathogenic lifestyle, yet are phylogenetically distinct from fungi, with brown algae and diatoms as their close relatives (Baldauf et al., 2000). As with other Oomycetes such as Pythium, Phytophthora can propagate sexually or asexually. Sexual reproduction leads to the formation of thick-walled oospores that may survive harsh environments. In constrast, asexual reproduction involves the formation of sporangia and biflagellated wall-less zoospores.

Sporangia of many Phytophthora species may germinate directly through a germ tube or indirectly by releasing zoospores. The released zoospores can swim in water film surrounding soil particles to find potential hosts via chemotaxis. After landing on the plant surface, they encyst rapidly and germinate. Subsequently, germ tubes differentiate to form appressoria and penetration pegs that penetrate into the plant tissue to assist pathogen colonization (Hardham, 2001). Therefore, zoospores play a central role in plant infection and pathogen dispersal and represent a critical component of the disease cycle of most oomycete pathogens. 
The formation of sporangia starts with differentiation of mycelia into sporangiophores, which are contiguous and similar in structure to vegetative hyphae (Hemmes, 1983). Subsequently, nuclear division occurs rapidly within the sporangiophores, followed by a quick flow of nuclei and cytoplasm into terminal swellings, which then develop into sporangia (Christen and Hohl, 1972; Maltese et al., 1995). Sporangium formation may be triggered by changes in environmental factors such as nutrients, light, humidity, and $\mathrm{pH}$ (Erwin and Ribeiro, 1996). In some Phytophthora spp., sporangia can form spontaneously as cultures age, whereas others require depletion of media nutrients to ensure mass production of sporangia (Erwin and Ribeiro, 1996). Completion of sporangia formation is marked by the formation of a basal plug on the bottom to delimit the multinucleate sporangium from the coenocytic hyphae and an apical papilla on the top, which is involved in sporangium germination and zoospore release (Maltese et al., 1995).

The formation of zoospores can be triggered by cool temperatures, a process known as "cold shock," which induces cytokinesis of the sporangial cytoplasm and subsequent compartment of a single nucleus into each zoospore (Ribeiro, 1983). Zoosporogenesis is accompanied by changes in cytoskeletal organization and vesicle distribution as well as movement of ions such as calcium (Hyde et al., 1991; Jackson and Hardham, 1996). In Phytophthora cinnamomi, cold shock elicited an instant increase in the concentration of calcium in the sporangial cytoplasm, which was followed by a second increase of calcium concentration during cytoplasmic cleavage to regulate cytokinesis (Jackson and Hardham, 1996). In $P$. infestans, zoosporogenesis was impaired by treatment with the calcium channel blocker verapamil, the calmodulin antagonist trifluoperazine, and the inositol trisphosphate (IP3)-receptorgated calcium channel antagonist 2-aminoethoxydiphenyl borate (Judelson and Roberts, 2002; Tani et al., 2004). These results suggest an important role for calcium signaling in zoosporogenesis of oomycetes.

Recent studies further demonstrated that zoosporogenesis involves major changes in expression profiles of many genes including those encoding putative protein kinases, transcription factors, ion channels and other regulator proteins, which are regulated via multiple signaling pathways (Kim and Judelson, 2003; Tani et al., 2004; Judelson et al., 2008, 2009). In addition, regulatory proteins that participate in sporangium formation and zoospore release of $P$. infestans include a protein kinase (Judelson and Roberts, 2002), the G-protein beta subunit (Latijnhouwers and Govers, 2003), the NIF transcriptional regulators (Judelson and Tani, 2007), and PiGK4, a G-protein-coupled receptor with a phosphatidylinositol phosphate kinase domain (Hua et al., 2013). Nonetheless, not much is known about the molecular players involved in calcium signaling in the asexual reproduction of Phytophthora.

Calcium signaling through the $\mathrm{Ca}^{2+}$-binding protein calmodulin and the $\mathrm{Ca}^{2+}$-calmodulin-dependent phosphatase calcineurin is an important pathway in many organisms including fungi, acting to modulate the process of budding, mating, stress response, and virulence (Thewes, 2014). In fungi, cellular calcium levels are regulated by multiple channels and transporters, with the low-affinity calcium uptake system (LACS) and high-affinity calcium uptake system (HACS) as two major calcium uptake pathways (Locke et al., 2000; Muller et al., 2001). When calcium availability is high, the primary calcium entry route is LACS, which is minimally composed of mating factor-induced gene 1 (FIG1), a transmembrane calcium channel and regulatory protein (Muller et al., 2003). In contrast, during low calcium availability, the major calcium entry route is HACS, which is composed of calcium channel homolog 1 (CCH1), mating pheromone-induced death 1 (MID1; Locke et al., 2000), and the PMP22_Claudin superfamily member Ecm7 (Martin et al., 2011; Ding et al., 2013). CCH1 encodes a transmembrane protein homologous to the $\alpha 1$-subunit of the L-type voltage-gated calcium channel in mammals (Fischer et al., 1997; Locke et al., 2000). In contrast, MID1 shows no overall sequence similarity to any known $\beta$-subunits of the L-type voltage-gated calcium channel (Iida et al., 1994). In Saccharomyces cerevisiae, deletion mutants of CCH1 or MID1 in a MATa background died shortly after exposure to $\alpha$-factor in a calcium-limited medium (Iida et al., 1994; Fischer et al., 1997). An Aspergillus nidulans mutant (midA) carrying a MID1 mutation had highly branched hyphae and showed at the hyphal apex an aberrant distribution pattern of the Spitzenkörper, the organizing center for hyphal growth and morphogenesis (Wang et al., 2012). As well, the CCH1-MID1 channel is indispensable for the survival of fungal pathogens such as Candida albicans and Cryptococcus neoformans in low-calcium environments (Locke et al., 2000; Bonilla et al., 2002; Liu et al., 2006), and also function to maintain calcium homeostasis in response to environmental and endoplasmic reticulum stress (Hong et al., 2010).

Phytophthora parasitica Dastur (= Phytophthora nicotianae Breda de Haan) causes root rot, foot rot, leaf blight, and fruit rot in a wide variety of crop species (Erwin and Ribeiro, 1996). During asexual reproduction, it produces terminally on the sporangiophores papillate, non-caducous, spherical to obturbinate sporangia, which may release zoospores after incubation at cool temperature. In this study, we demonstrated that the expression of PpMID1, an MID1 homolog of $P$. parasitica, is highly induced in $P$. parasitica sporangia and silencing PpMID1 causes severe defects in the asexual reproduction of the pathogen, including the formation of aberrant sporangia and inability to produce zoospores, as well as a reduction in virulence. These results indicate an important role for $P$ PMID1 in the asexual development and virulence of $P$. parasitica.

\section{MATERIALS AND METHODS}

\section{Growth of $P$. parasitica and Plant Material}

Phytophthora parasitica isolates (731, 991, and 94069) were provided by P. J. Ann (Taiwan Agricultural Research Institute, WuFeng, Taiwan). For routine culture, they were grown on $20 \%$ V8 juice agar (20\% Campbell's V8 juice, $0.02 \% \mathrm{CaCO}_{3}$, and $2 \%$ agar) at $25^{\circ} \mathrm{C}$ in the dark. Nicotiana benthamiana was grown in a mixture of peat moss, perlite, and vermiculite at $25^{\circ} \mathrm{C}$ under a 12-h light/dark regime. 


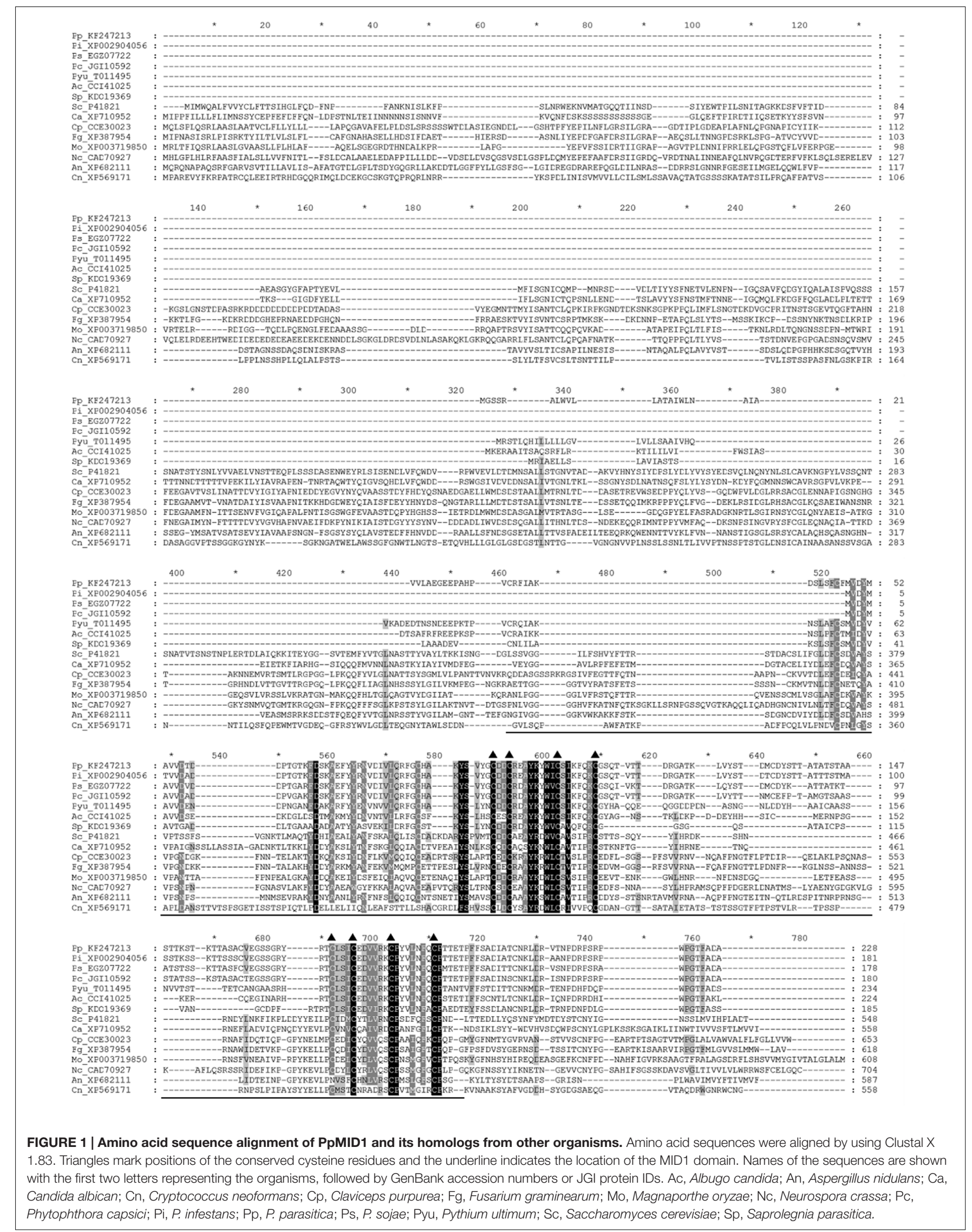




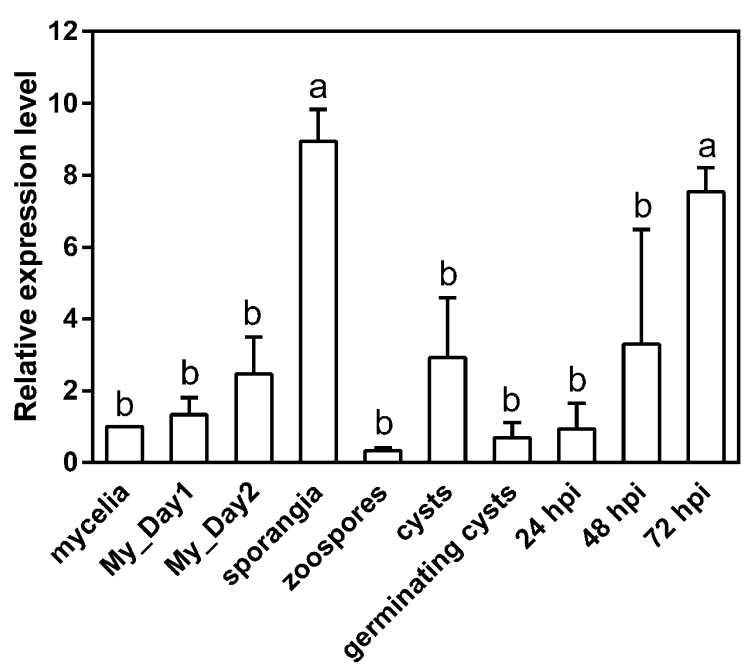

FIGURE 2 | Expression of PpMID1 is highly induced in the sporangia of Phytophthora parasitica and in planta. GRT-PCR of total RNA from different life stages of $P$. parasitica and 5-week-old Nicotiana benthamiana leaves inoculated with $P$. parasitica. Raw data were normalized to the transcript level of WS21 as an internal control and shown as fold change relative to that of mycelium. Data are mean \pm SD from 3 independent experiments. Different letters indicate significant difference by Fisher's least significant difference test $(P<0.05)$. "My_Day1" and "My_Day2" indicate mycelia harvested at 1 and 2 days, respectively, after light treatment for inducing sporangium formation. hpi: hours post-inoculation.

\section{Molecular Cloning and Sequence Analysis of PpMID1}

The genomic and cDNA sequences of PPMID1 were amplified by PCR and reverse transcriptase PCR, respectively, with

the primers Ppmid1_795F and Ppmid1_821R (Supplementary Table S1) and cloned into pGEM-T Easy vector (Promega, Fitchburg, WI, USA). Following sequence analysis, conserved protein domains were predicted by use of the InterProScan 5 server ${ }^{1}$. Alignment of P PMID1 and its homologs from other organisms involved use of Clustal X. Phylogenetic trees were generated by the neighbor-joining algorithm implemented in MEGA 6.06 with the default parameters. Nodal support of the tree was estimated by bootstrapping with 1,000 pseudoreplicate data sets.

\section{Isolation of RNA}

Total RNA from different life stages of $P$. parasitica, including mycelia, sporangia, zoospores, cysts, and germinating cysts were prepared with use of TriZol reagent (Invitrogen-Life Technologies, Carlsbad, CA, USA) as described by Yan and Liou (2006). RNA from inoculated plants was isolated by using the Plant Total RNA Extraction Kit (Viogene-BioTek, New Taipei City, Taiwan).

\section{Pathogen Inoculation}

For inoculation, the P. parasitica isolate was grown in $20 \% \mathrm{~V} 8$ juice agar for 5 days. Then, mycelial disks ( $5 \mathrm{~mm}$ id.) were excised from the margin and used to inoculate detached 4 th or 5 th leaves of 5-week-old $N$. benthamiana plants. The inoculated leaves were kept in a plastic box with high humidity at $25^{\circ} \mathrm{C}$ in the dark.

\section{Quantitative RT-PCR}

Residual DNA present in the RNA solution was removed by use of the Turbo-DNA free kit (Ambion, Huntingdon, UK). First-strand cDNA was synthesized by using SuperScript III reverse transcriptase (Invitrogen-Life Technologies), with $1 \mu \mathrm{g}$

\footnotetext{
${ }^{1}$ http://www.ebi.ac.uk/Tools/pfa/iprscan5/
}
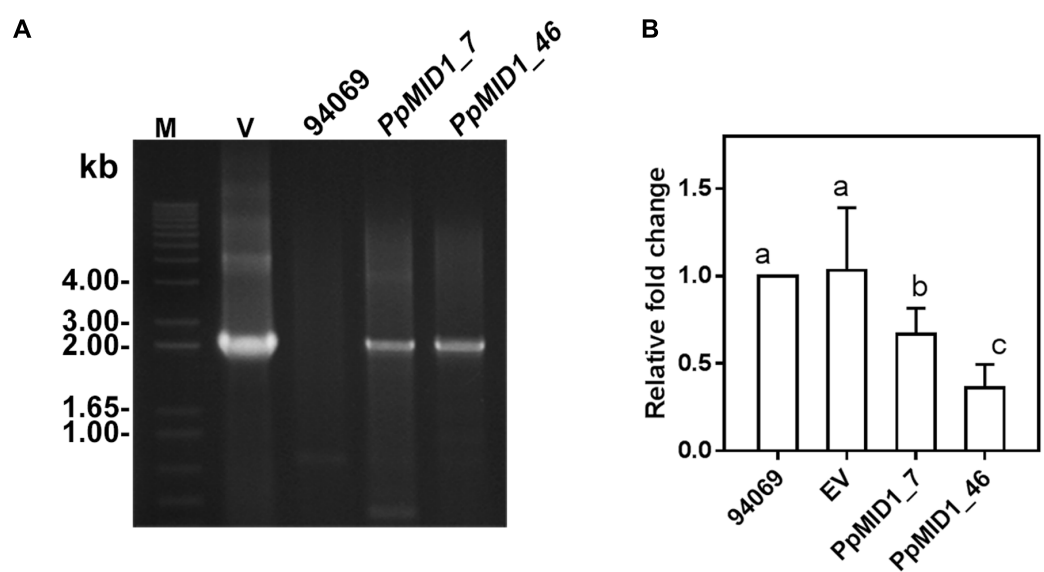

FIGURE 3 | Analyses based on PCR and qRT-PCR show both PpMID1_7 and PpMID1_46 as Phytophthora parasitica transformants. (A) PCR analysis. Genomic DNAs extracted from the PpMID1-silenced transformants (PpMID1_7 and PpMID1_46) and wild-type P. parasitica (94069) were amplified with the primers nptF1 and transR2. V, plasmid DNA as the PCR template; M, size marker (in kb). (B) The expression of PpMID1 is downregulated in the mycelium of PpMID1-silenced transformants. qRT-PCR analysis of total RNA from the mycelia of PpMID1_7 and PpMID1_46, the empty vector transformant (EV), and the wild type (94069). Raw data were normalized by transcripts of WS21 as an internal control and shown as fold change relative to that of the wild-type strain. Data are mean \pm SD from 3 independent experiments. Different letters indicate significant difference by Fisher's least significant difference test $(P<0.05)$. 
TABLE 1 | PpMID1-silenced transformants produce less sporangia and branch more frequently.

\begin{tabular}{|c|c|c|c|c|}
\hline Phytophthora parasitica & Growth rate $(\mathrm{mm} / \mathrm{d})^{1,5}$ & No. of sporangia 2,5 & No. of zoospores ${ }^{3,5}$ & Branch-to-branch hyphal interval $(\mu \mathrm{m})^{4,5}$ \\
\hline 94069 & $14.2 \pm 1.4^{a}$ & $967 \pm 53^{a}$ & $755 \pm 79^{a}$ & $422.2 \pm 46.2^{a}$ \\
\hline EV & $15.4 \pm 0.1^{a}$ & $878 \pm 72^{a}$ & $873 \pm 60^{a}$ & $365.2 \pm 48.9^{a}$ \\
\hline PpMID1_7 & $12.8 \pm 2.5^{a}$ & $446 \pm 31^{b}$ & $0 \pm 0^{b}$ & $148.9 \pm 24.9^{b}$ \\
\hline PpMID1_46 & $10.9 \pm 1.1^{a}$ & $600 \pm 93^{b}$ & $6 \pm 1^{b}$ & $114.0 \pm 25.7^{b}$ \\
\hline
\end{tabular}

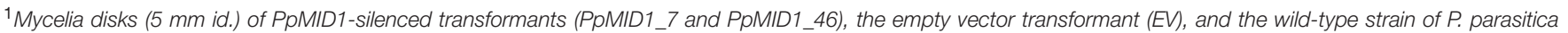

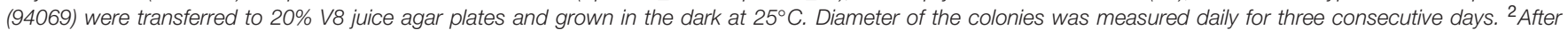

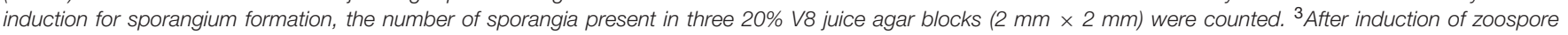
formation by cold shock $\left(19^{\circ} \mathrm{C}, 30 \mathrm{~min}\right)$ and release of zoospores from sporangia, the numbers of zoospores present in $10 \mu \mathrm{L}$ induction solution were determined. ${ }^{4} \mathrm{P}$. parasitica was grown in the same way as described in '1'. For better observation, mycelia disks (5 mm id.) excised at day 5 were immersed in $60 \mu \mathrm{L} 20 \%$ V8 juice medium and pictures were taken under a microscope 3 days later. For each isolate, six branch intervals were measured by using ImageJ. ${ }^{5}$ Data are mean \pm SD from 3 independent experiments; means followed by different letters indicate significant difference by Fisher's least significant difference test (P < 0.05$)$.

RNA as the template and $25 \mu \mathrm{M}$ VdT (V: A, C, or G at the $\left.3^{\prime}\right)$ as the primer. Quantitative PCR involved the Step One Plus Real-Time PCR System (Applied Biosystems, Foster City, CA, USA). The reaction mixture $(20 \mu \mathrm{L})$ contained $10 \mu \mathrm{L}$ Power SYBR Green PCR Master Mix (Applied Biosystems), $0.5 \mu \mathrm{M}$ primers (Supplementary Table S1), and $1 \mu \mathrm{L}$ cDNA product diluted 10 times. The settings for PCR were $95^{\circ} \mathrm{C} / 10 \mathrm{~min}, 42$ cycles of $95^{\circ} \mathrm{C} / 15 \mathrm{~s}, 60^{\circ} \mathrm{C} / 1 \mathrm{~min}$, followed by melting curve analysis. For analysis of gene expression in $P$. parasitica, the level of WS21, which encodes a ribosomal protein, was an internal control for normalization (Yan and Liou, 2006).

\section{Construction of the Silencing Vector}

With pHAM34 (a gift from Dr. Y. C. Wang, Department of Plant Pathology, Nanjing Agricultural University, Nanjing, China) as the template, a DNA fragment containing 5'HAM34 and 3'HAM34 (Judelson and Michelmore, 1991) was amplified by using pro_ham34_HindIII_F.2/ter_ham34_XbaI_R as primers and subcloned into the HindIII and XbaI sites of pTH209 (a gift from Dr. H. Judelson, Department of Plant Pathology and Microbiology, University of California, Riverside, CA) to generate pEX (Supplementary Figure S1). Subsequently, an intron sequence of the tomato NIPRa gene (Liou et al., unpublished data) was amplified by using primers pHP_intron3_F and pHP_intron3_R (Supplementary Table S1), with the addition of three different restriction sites on either end, namely NheI, AvrII, and SacII sites at the $5^{\prime}$ end and SacI, SpeI, and BamHI sites at the $3^{\prime}$ end. This DNA fragment was then inserted into the SmaI site of pEX to generate pHP. For construction of pHPdest, two Gateway destination cassettes provided by the Gateway vector conversion system (Invitrogen-Life Technologies) were ligated into $\mathrm{pHP}$ in an opposite orientation, accomplished by use of NheI and SacII sites at the $5^{\prime}$ end and SacI and BamHI sites on the $3^{\prime}$ end of the intron sequence. To construct the silencing vector, the partial sequence of PpMID1 was amplified with the primers PhyMID1_F and PhyMID1_R (Supplementary Table S1) and subcloned into pENTR/D-TOPO vector (Invitrogen-Life Technologies). LR recombination reaction then involved use of pHPdest and the Gateway LR clonase II enzyme mix (Invitrogen-Life Technologies) to obtain the silencing vector pHPdest::PpMID1.

\section{Preparation of Protoplasts and Polyethylene Glycerol (PEG)-mediated Transformation}

Protoplasts of $P$. parasitica were prepared by using young mycelial mats as described (McLeod et al., 2008), followed by transformation of the protoplasts with some modifications. Three days after growth of $P$. parasitica in $20 \%$ V8 juice medium (20\% Campbell's V8 juice and $0.02 \% \mathrm{CaCO}_{3}$ ), the mycelia were harvested by filtration and washed in $0.4 \mathrm{M}$ mannitol to weed out residual medium. The mycelia (counted as one volume) were digested at $25^{\circ} \mathrm{C}$ for $45 \mathrm{~min}$ in 14 volumes of a digestion solution [0.5\% (w/v) cellulase (Sigma-Aldrich), 1.5\% (w/v) lysing enzyme (L1412, Sigma-Aldrich), 0.4 M mannitol, $20 \mathrm{mM} \mathrm{KCl,} 10 \mathrm{mM}$ $\mathrm{CaCl}_{2}$, and $20 \mathrm{mM} \mathrm{MES}, \mathrm{pH}$ 5.7]. Then the digested solution was filtrated through 4 layers of Miracloth (EMD Millipore, Billerica, MA, USA), and protoplasts were collected by centrifugation for 3 min with $1,000 \mathrm{~g}$ at $25^{\circ} \mathrm{C}$. After a rinse with $25 \mathrm{~mL}$ W5 solution (154 $\mathrm{mM} \mathrm{NaCl}, 125 \mathrm{mM} \mathrm{CaCl}_{2}, 5 \mathrm{mM} \mathrm{KCl}$, and $2 \mathrm{mM} \mathrm{MES}$, $\mathrm{pH}$ 5.7), protoplasts were resuspended in $5 \mathrm{~mL} \mathrm{~W} 5$ solution and incubated on ice for $30 \mathrm{~min}$, then collected by centrifugation at $1000 \mathrm{~g}$ for $3 \mathrm{~min}$, followed by resuspension in the $\mathrm{MMg}$ solution (0.4 M mannitol, $15 \mathrm{mM} \mathrm{MgCl}_{2}$, and $4 \mathrm{mM} \mathrm{MES}$, $\mathrm{pH}$ 5.7) to a final concentration of $1 \times 10^{6}$ protoplasts $/ \mathrm{mL}$ and incubation at room temperature for $10 \mathrm{~min}$. For PEG-mediated transformation, 1-mL aliquots of the protoplast suspension were mixed with $30-50 \mu \mathrm{g}$ of plasmid DNA in 15-mL Falcon tubes, then incubated on ice for $10 \mathrm{~min}$. Subsequently, $580 \mu \mathrm{L}$ of PEG 4000 solution [40\% (v/v) PEG 4000 (Sigma-Aldrich), $0.2 \mathrm{M}$ mannitol, and $0.1 \mathrm{M} \mathrm{CaCl}_{2}$ ] was added slowly to each Falcon tube and the tube was gently inverted several times to mix the contents thoroughly. This process was repeated twice at an interval of $30 \mathrm{~s}$ to add a total of $1.74 \mathrm{~mL}$ PEG 4000 solution. The mixture was then incubated on ice for $20 \mathrm{~min}$, followed by the addition of $2 \mathrm{~mL} \mathrm{V8-M} \mathrm{medium} \mathrm{(20 \%} \mathrm{V8} \mathrm{juice,} 0.5 \mathrm{M}$ mannitol, and $10 \mathrm{mM} \mathrm{CaCl}_{2}$ ) and $2 \mathrm{~min}$ later $8 \mathrm{~mL} \mathrm{V8-M} \mathrm{medium.} \mathrm{Finally,}$ the mixture was incubated at $25^{\circ} \mathrm{C}$ for $7 \mathrm{~h}$ to allow protoplasts to regenerate, with the addition of $50 \mathrm{ppm}$ ampicillin to prevent bacterial contamination.

After regeneration, protoplasts were examined under a microscope for their recovery. Once the regenerated protoplasts began to germinate, the cells were suspended in $20 \%$ V8 juice medium and plated on $20 \%$ V8 agar plates that contained $10 \mathrm{ppm}$ 

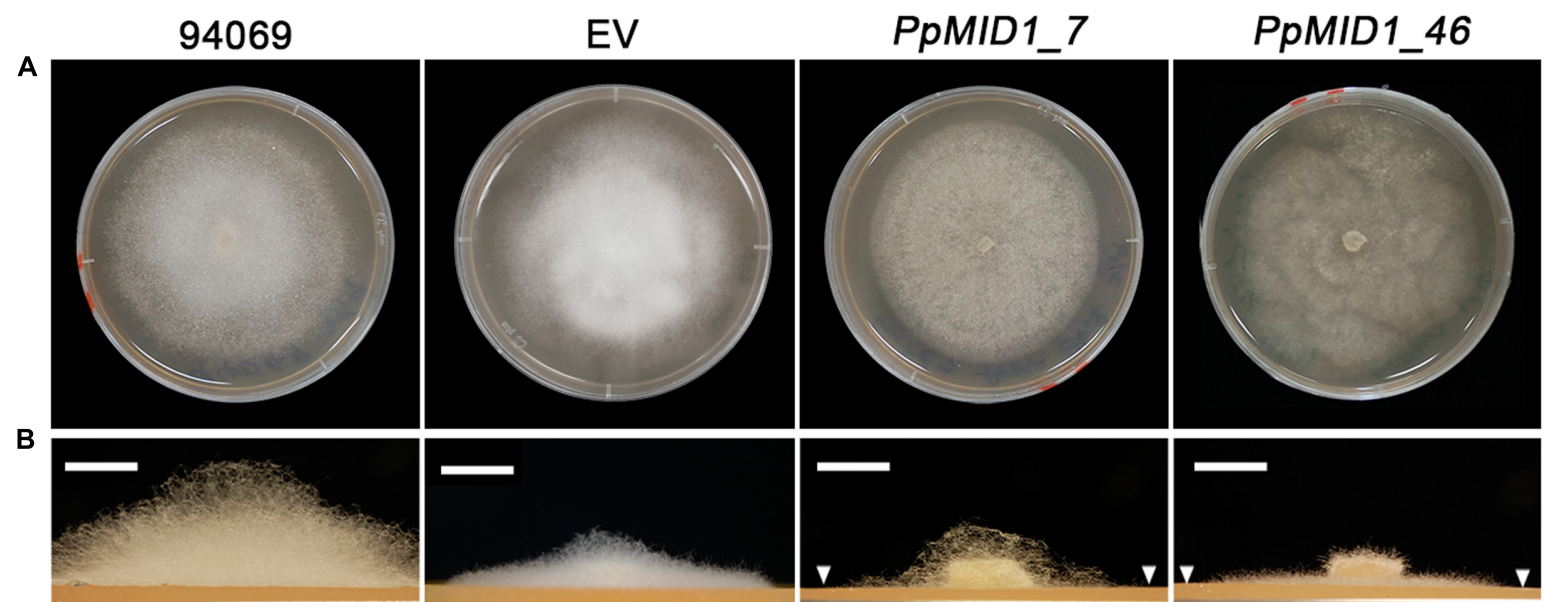

C
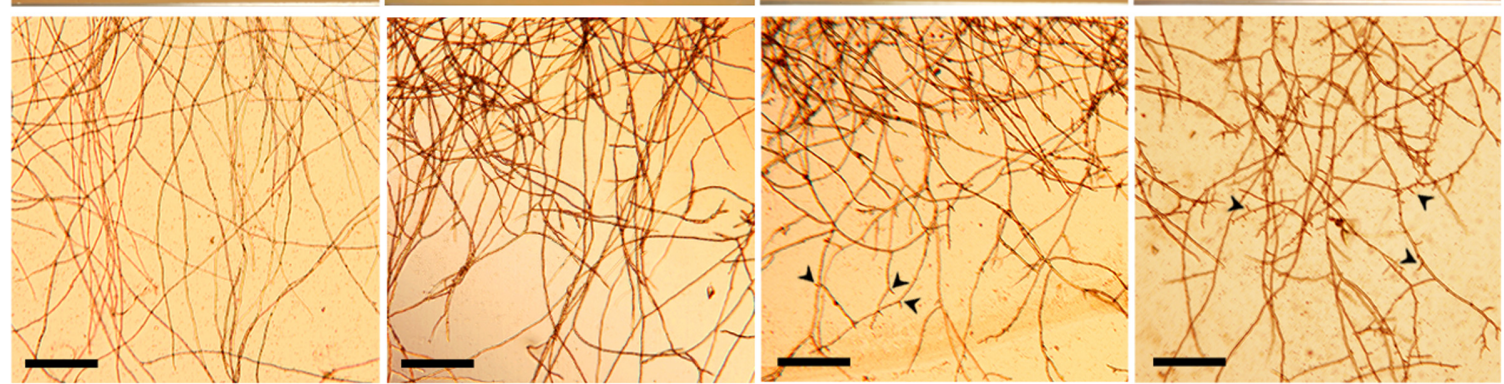

FIGURE 4 | PpMID1-silenced transformants produce less aerial mycelia and mycelia in liquid culture are highly branched. (A) Colony morphology. Photographs were taken 7 days after subculture. (B) PpMID1-silenced transformants produced less aerial mycelia on solid medium. Mycelia disks (5 mm id.) of silenced transformants (PpMID1_7 and PpMID1_46), EV, and wild-type Phytophthora parasitica (94069) were transferred to 20\% V8 juice agar and grown in the dark at $25^{\circ} \mathrm{C}$. Photographs for the side view of each colony were taken 3 days after subculture. Arrowheads, colony margins of silenced transformants. Bar $=5 \mathrm{~mm}$. (C) Mycelia produced by PpMID1-silenced transformants in liquid medium are highly branched. Mycelia disks (5 mm id.) of PpMID1_7, PpMID1 46, EV, and the wild type (94069) were transferred to $20 \%$ V8 juice medium and grown at $25^{\circ} \mathrm{C}$ in the dark. Photographs were taken under a microscope 1 day later. Arrowheads, branched hyphae. Bar $=100 \mu \mathrm{m}$.

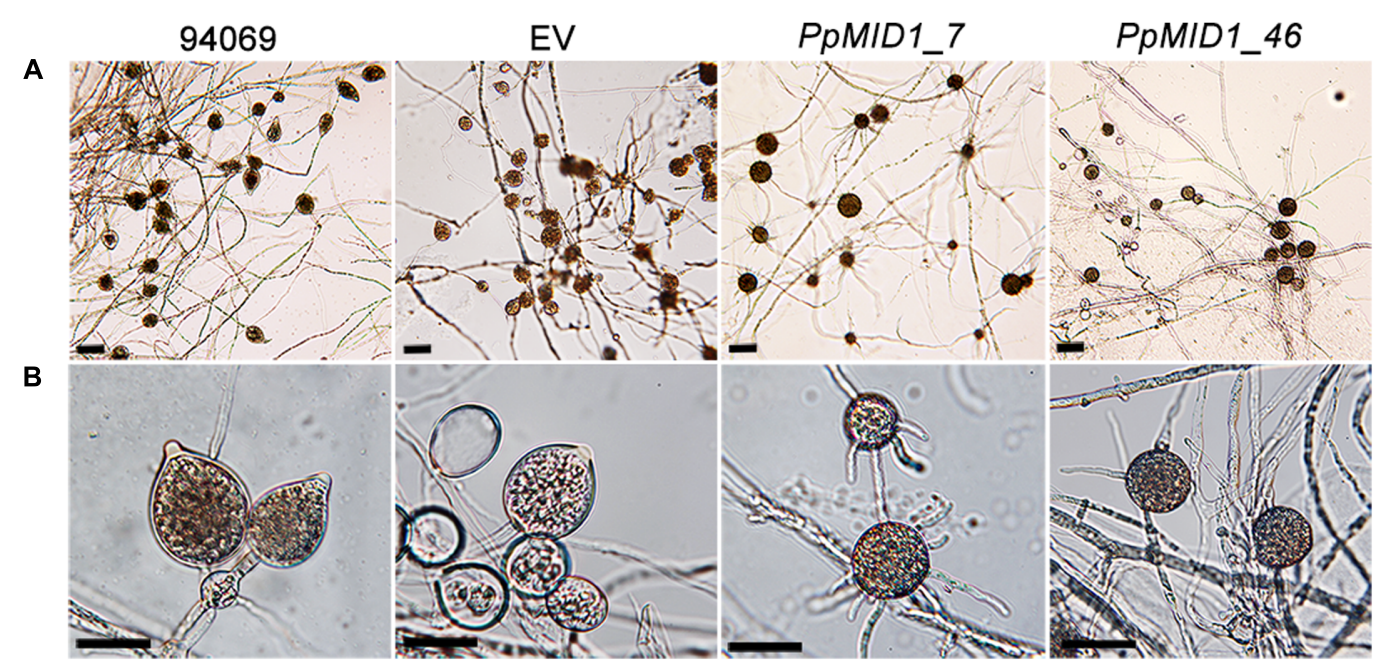

FIGURE 5 | Sporangia produced by PpMID1-silenced transformants show aberrant morphology. Mycelial blocks of PpMID1_7 and PpMID1_46, EV, and wild-type Phytophthora parasitica (94069) were incubated in $1 \%$ V8 juice medium under light at $25^{\circ} \mathrm{C}$ to induce sporangium formation. After 3 days, photographs were taken under a microscope. (A) Formation of sporangia; (B) Four-fold magnification of (A). Bar $=25 \mu \mathrm{m}$. 

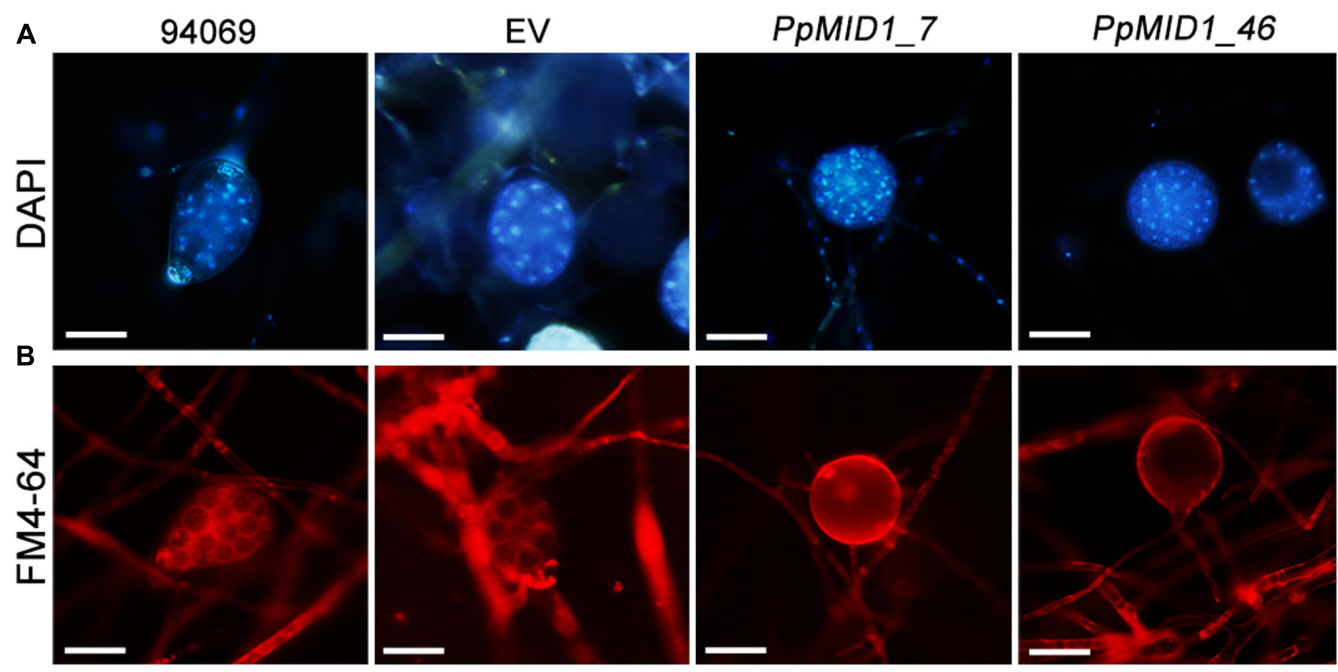

FIGURE 6 | Sporangia of PpMID1-silenced transformants show defective cytoplasmic cleavage. Mycelia blocks of PpMID1_ 7 and PpMID1_46, EV, and wild-type Phytophthora parasitica (94069) were incubated in $1 \%$ V8 juice medium under light at $25^{\circ} \mathrm{C}$ to induce sporangium formation. After 3 days, the sporangia were cold-shocked at $19^{\circ} \mathrm{C}$ for 30 min to trigger zoospore differentiation, then stained with: (A) DAPI for nuclear division; (B) lipophilic dye FM 4-64 to examine the occurrence of cytoplasmic cleavage. Bar $=20 \mu \mathrm{m}$.

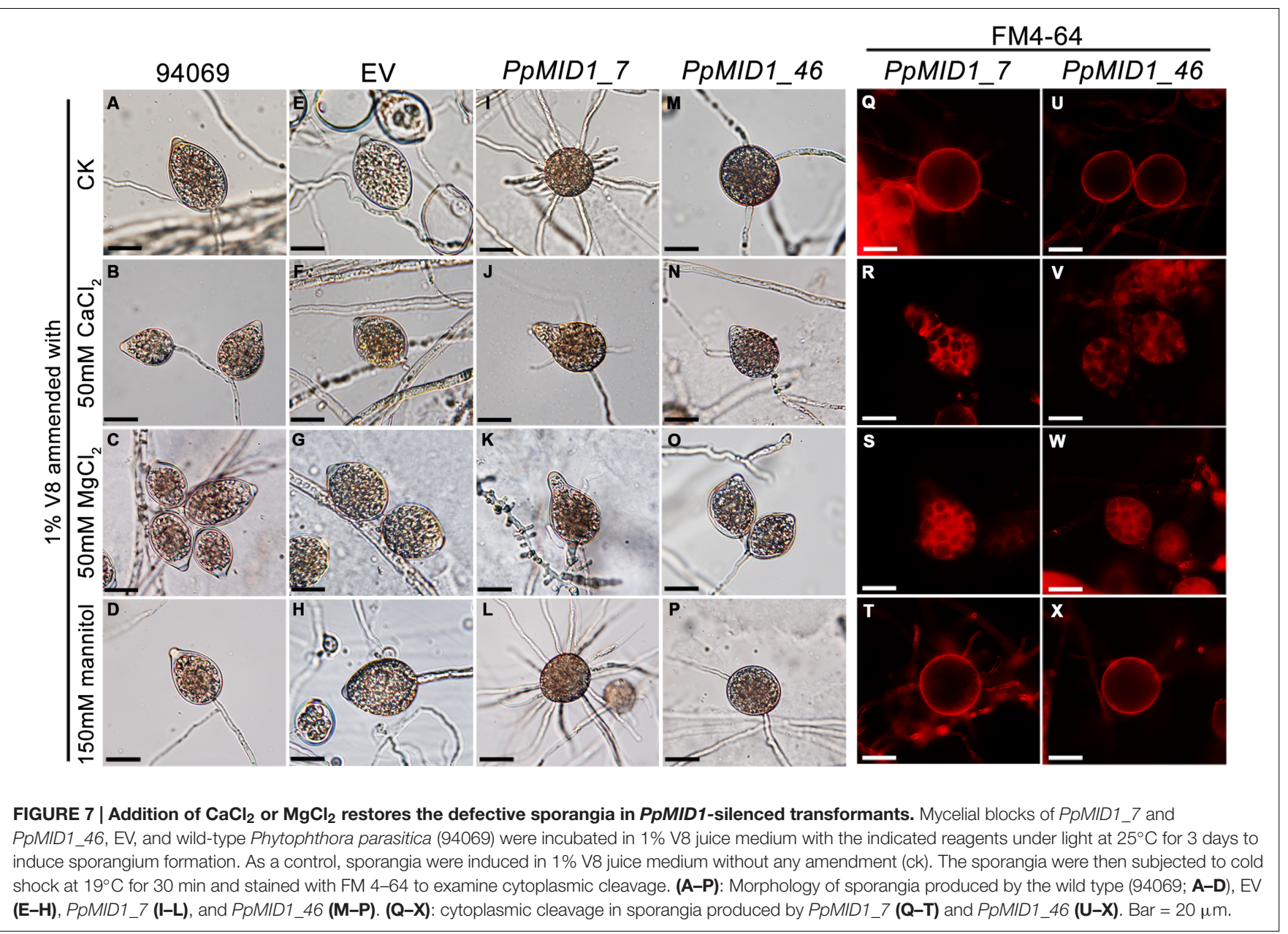


TABLE 2 | Morphology of sporangia produced by PpMID1-silenced transformants is reversed with the addition of calcium or magnesium ions.

\begin{tabular}{|c|c|c|c|}
\hline \multirow[b]{2}{*}{ Transformant } & \multicolumn{3}{|c|}{$\begin{array}{l}\text { Percentage of recovered sporangia in } 1 \% \text { V8 juice } \\
\text { medium containing }{ }^{1}\end{array}$} \\
\hline & $50 \mathrm{mM} \mathrm{CaCl} 2$ & $50 \mathrm{mM} \mathrm{MgCl} 2$ & $150 \mathrm{mM}$ mannitol \\
\hline PpMID1_7 & $14.8 \pm 5.1$ & $32.5 \pm 3.9$ & $0.3 \pm 0.4$ \\
\hline PpMID1_46 & $24.1 \pm 2.5$ & $20.6 \pm 6.0$ & $2.0 \pm 0.5$ \\
\hline
\end{tabular}

${ }^{1}$ Mycelia blocks (2 mm $\times 2 \mathrm{~mm}$ ) of the PpMID1-silenced transformants (PpMID1_7 and PpMID1_46) were incubated in 1\% V8 juice medium amended with indicated ingredients. Following induction of sporangium formation, the number of all sporangia formed in three mycelia blocks was counted. Sporangia bearing a papilla were considered "recovered in morphology" and shown as percentage of the total number of sporangia. Data are mean $\pm S D$ from 3 independent experiments.

geneticin. After incubation at $25^{\circ} \mathrm{C}$ in the dark for 4 days, colonies appearing on the agar plate were transferred to fresh $20 \% \mathrm{~V} 8$ agar plates amended with $10 \mathrm{ppm}$ geneticin for a second round of selection. Only transformants passing at least two rounds of geneticin selection underwent further analysis by PCR primed with nptF1 and transR2, which target the backbone of the silencing vector.

\section{Recovery of Silenced Sporangia}

To induce sporangium formation, mycelial blocks of $P$. parasitica $(2 \mathrm{~mm} \times 2 \mathrm{~mm})$ excised from a 7-day-old culture were immersed in $1 \%$ V8 juice medium (a 20x dilution of the $20 \%$ V8 juice medium) alone or amended with the test solution: $50 \mathrm{mM} \mathrm{CaCl}$, $50 \mathrm{mM} \mathrm{MgCl}_{2}$, or $150 \mathrm{mM}$ mannitol. The osmotic pressure of each solution was adjusted to the same level according to the van't Hoff law $(\pi=i C R T)$. After incubation at $25^{\circ} \mathrm{C}$ under white light for 3 days, the mycelial blocks were examined under a microscope for the formation of sporangia.

\section{Staining of Sporangia with DAPI and FM 4-64}

Sporangia were first stained with DAPI (4', 6-diamidino-2phenylindole; Invitrogen-Life Technologies), then FM 4-64 (Invitrogen-Life Technologies) as described (Zhang et al., 2012) with some modifications. Briefly, sporulating mycelial blocks were incubated in a DAPI solution [300 nM in Hank's balanced salt solution (HBSS)] for $10 \mathrm{~min}$ and the residual DAPI was washed out with HBSS. Then FM 4-64 solution $(5 \mu \mathrm{g} / \mathrm{mL}$ in HBSS) was added, followed by immediate examination of sporangia under a Leica DMLB microscope (Buffalo Grove, IL) equipped with filter cube A (BP 340-380 nm, LP $425 \mathrm{~nm}$; for DAPI) or I3 (BP 450-490 nm, LP $515 \mathrm{~nm}$; for FM 4-64). Images were captured by using a Canon (Ohta-ku, Tokyo, Japan) digital camera EOS 550D.

\section{The Mating Experiment}

Mycelia blocks ( $5 \mathrm{~mm} \times 5 \mathrm{~mm}$ ) of test isolates of $P$. parasitica were placed pairwise on the glass slides inside a Petri dish. After incubation at $25^{\circ} \mathrm{C}$ in the dark for 7 days, the morphology of oospores was examined and their number was counted under a microscope.

\section{Trypan Blue Staining}

Trypan blue staining was performed as described (Wilson and Coffey, 1980). N. benthamiana leaves were boiled in $96 \%$ ethanol and then for $45 \mathrm{~s}$ in a trypan blue solution prepared by mixing two volumes of $96 \%$ ethanol with one volume of a solution containing $0.03 \%(\mathrm{w} / \mathrm{v})$ trypan blue (Fluka, Buchs, Switzerland) in lactophenol (lactic acid:phenol:glycerol: $\mathrm{ddH}_{2} \mathrm{O}=1: 1: 1: 1$ ). The stained leaves were then rinsed several times with $96 \%$ ethanol until the background was clear.

\section{Statistical Analysis}

Statistically significant differences were analyzed by Fisher's least significant difference test. Significance was set at $\mathrm{P}<0.05$ and determined by use of PASW Statistics 18 (SPSS Inc, Chicago, IL, USA).

\section{RESULTS}

\section{Sequence Analysis of PpMID1}

To identify $P$. parasitica gene(s) homologous to $M I D 1$, we performed a BLAST search of the $P$. parasitica (INRA-310) genome database of the Broad Institute, with $S$. cerevisiae MID1 (GAA25818.1) used as a query and found a homolog, PPTG_18661 [P. parasitica INRA-310 (V2)], that contains an intron and encodes a protein of 228 amino acid residues (aa) with $26 \%$ identity with yeast MID1. We then cloned the genomic and cDNA sequence of this gene, hereafter named PpMID1 (GenBank accession no. KF247213), and found the sequences identical to that of PPTG_18661. InterProScan analysis revealed the presence of an N-terminal signal peptide (aa 1-25) as well as two signature matches: aa 35-195 for IPR024338 (stretch-activated cation channel MID1) (Figure 1) and aa 48-120 for IPR029604 (transmembrane protein FAM155, functionally uncharacterized). Notably, PpMID1 is much smaller than fungal MID1, with conservation confined to the carboxyl terminus, especially the 9 cysteine residues at specific positions (Figure 1, triangles), which are essential for the function of MID1 in S. cerevisiae (Maruoka et al., 2002). Homologs of MID1 were identified in the genome databases of other oomycetes, including $P$. capsici, $P$. infestans, $P$. sojae, Pythium ultimum, Albugo candida, and Saprolegnia parasitica. Phylogenetic analysis indicated that the MID1 homologs from Phytophthora spp. formed a cluster with the other three oomycete pathogens but distinct from MID1 homologs from true fungi (Supplementary Figure S2).

\section{Expression of PpMID1 Is Highly Induced in Sporangia and In Planta}

To examine the expression pattern of PpMID1, we prepared total RNA from different life stages of $P$. parasitica for quantitative RTPCR analysis. The data were normalized to transcript levels of WS21 as an internal control and expressed as fold change relative to the level in mycelium. WS21 encodes a ribosomal protein and shows constant expression throughout different life stages of $P$. parasitica (Yan and Liou, 2006). When the mycelia were subjected to light treatment to induce sporangium formation, the 


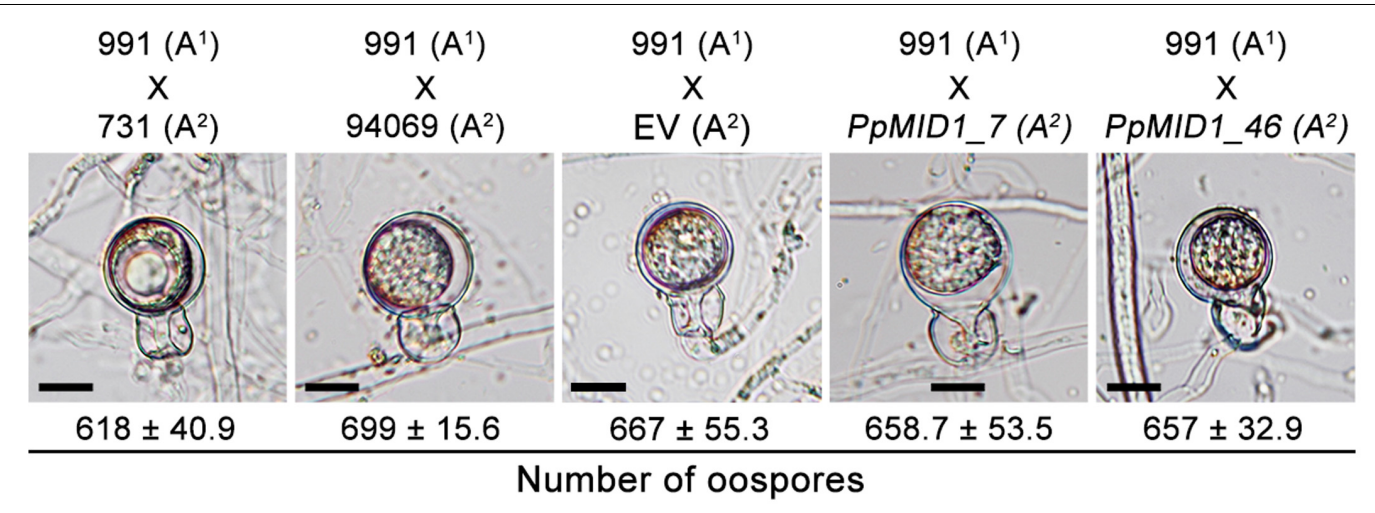

FIGURE 8 | Silencing of PpMID1 has no effect on oospore formation. Pairwise testing of PpMID1_7, PpMID1_46, EV, and wild-type strains of Phytophthora parasitica $\left(94069,991\right.$, and 731 ) for their ability to mate and produce oospores. Each paired agar culture was incubated at $25^{\circ} \mathrm{C}$ in the dark for 7 days, then examined under a microscope for oospore formation. Numbers under each photograph indicate the mean \pm SD number of oospores present in $5 \times 5$ mm ${ }^{2}$ agar blocks from 3 independent experiments $(n=3)$. Bar $=12.5 \mu \mathrm{m}$.

expression of PpMID1 increased gradually from 1 to 2 days after light treatment (Figure 2, My_Day1 and My_Day2), with a peak for sporangia collected at 3 days after light treatment (Figure 2). We also analyzed the expression of PPMID1 in N. benthamiana leaves inoculated with $P$. parasitica. Compared to the transcript level detected in germinating cysts, PPMID1 was slightly induced at $48 \mathrm{~h}$ post-inoculation (hpi) but highly induced at $72 \mathrm{hpi}$, when abundant sporangia formed on the infected leaves (Figure 2). These results suggest an important role of PpMID1 during the formation of sporangia in P. parasitica.

\section{Generation of PpMID1-silenced Transformants}

To characterize the function of PpMID1, we generated PpMID1-silenced transformants by double-stranded RNAmediated gene silencing with pHPdest::PpMID1 used as the silencing vector (Supplementary Figure S1). pHPdest was constructed by using pTH209 (Judelson et al., 1991) as the backbone to allow generation of the silencing vectors by use of the Gateway cloning system. The strategy for constructing pHPdest and the silencing vector is detailed in Materials and methods. After transformation of $P$. parasitica protoplasts with the silencing vector pHPdest::PPMID1 and regeneration, we obtained a total of 540 putative transformants based on geneticin resistance. Most of them died after several rounds of subculture on V8 medium amended with geneticin. The remaining 48 isolates were further examined for changes in phenotypes, especially formation of sporangia and their characteristics, and analyzed by PCR primed with nptF1 and transR2 (Supplementary Table S1). Nonetheless, 46 isolates, likely transient transformants, showed geneticin resistance for around 6 months and were unable to grow in the V8 medium amended with geneticin in the subsequent subculture. Only PpMID1_7 and PpMID1_46 turned out to be stable transformants. Meanwhile, we obtained several $P$. parasitica transformants carrying the empty pHPdest vector as a control. Both PpMID1_7 and PpMID1_46 showed generated amplicons of the expected size (Figure $3 \mathbf{A}$ ) and significantly lower expression of PpMID1 in the mycelia than the wild-type $P$. parasitica and the empty vector transformants (EV) as revealed by qRT-PCR (Figure 3B). Expression of PpMID1 in the sporangia was not analyzed because of the small number of sporangia produced by the silenced transformants.

\section{PpMID1-silenced Transformants Show Defects in Mycelia Morphology and Produce Abnormal Sporangia}

To investigate the role of PpMID1 in different life stages of $P$. parasitica, we examined the growth rate and morphology of mycelium in the PpMID1-silenced transformants PpMID1_7 and PpMID1_46. When grown in $20 \%$ V8 juice agar at $25^{\circ} \mathrm{C}$ for 3 days, the growth of PpMID1_7 was similar to that of the wild-type strain (94069) and EV control, as revealed by colony diameters (Table 1). Interestingly, PpMID1_7 and PpMID1_46 produced much less aerial mycelia than the wild type and $\mathrm{EV}$ at 3 and 7 days after subculture (Figures $\mathbf{4 A}, \mathbf{B}$ ). Young hyphae produced by PpMID1_7 and PPMID1_46 tended to branch more frequently than did the wild type and $\mathrm{EV}$ (Figure 4C), with an average branch-to-branch interval of $148.9 \pm 24.9$ and $114.0 \pm 25.7 \mu \mathrm{m}$ for PpMID1_7 and PpMID1_46, respectively, as compared with $422.2 \pm 46.2 \mu \mathrm{m}$ for the wild type and $365.2 \pm 48.9 \mu \mathrm{m}$ for EV (Table 1). As well, branches of hyphae produced by PpMID1-silenced transformants were relatively short (Figure 4C), probably due to impeded hyphal growth after branching. These results suggest the involvement of PpMID1 in hyphal branching and tip growth.

To determine whether PPMID1 is involved in the formation of sporangia, we examined the number and morphology of sporangia produced by the transformants. The wildtype $P$. parasitica produced papillate, ovoid, and vesiculogencontaining sporangia, from 20 to $25 \mu \mathrm{m}$ in diameter (Figure 5), with a mean of $967 \pm 53$ per 2 -mm-square agar block. The EV control produced a similar amount of sporangia, $878 \pm 72$ per 2-mm-square agar block (Table 1), which were papillate, 


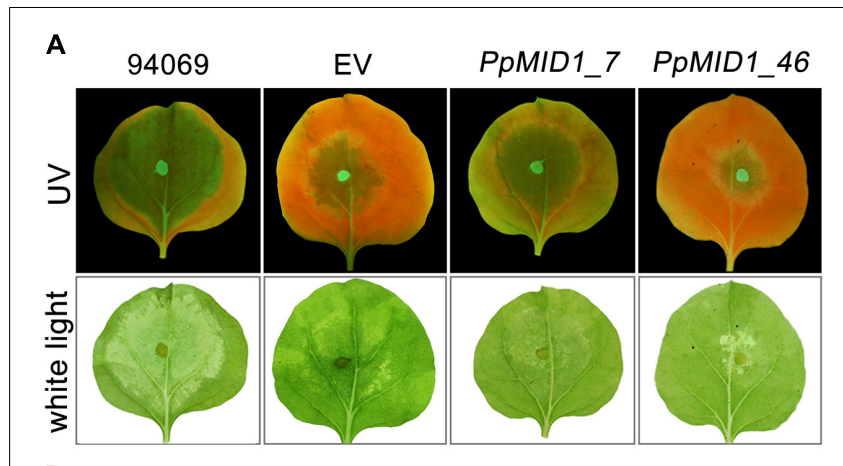

B

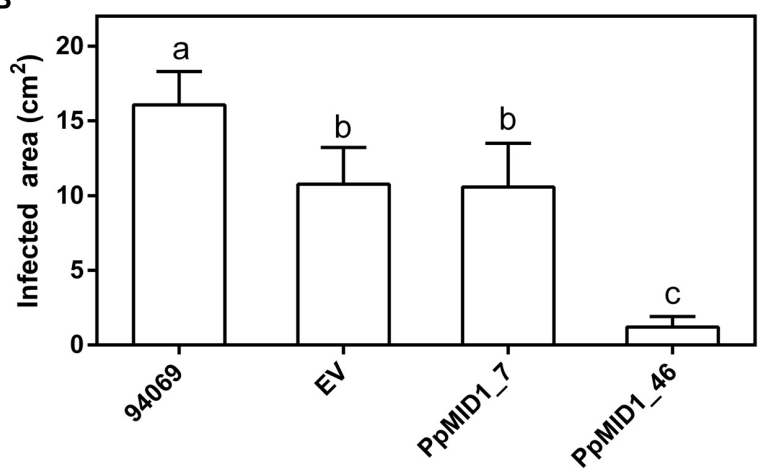

FIGURE 9 | PpMID1-silenced transformants show reduced virulence on plants. (A) Disease symptoms on Nicotiana benthamiana leaves caused by Phytophthora parasitica infection. For inoculation, mycelia disks (5 mm id.) of PpMID1_7 and PpMID1_46, EV, or wild-type P. parasitica (94069) were transferred to the center of leaves from 5 -week-old $N$. benthamiana. After incubation in a moisture box at $25^{\circ} \mathrm{C}$ for $72 \mathrm{~h}$, disease symptoms were examined and photographed under ultraviolet light (UV) or white light. (B) Quantification of infected areas on the inoculated N. benthamiana leaves. Infected areas on the inoculated leaves shown in (A) under UV light were measured by using ImageJ. Data are mean \pm SD from 4 independent experiments (each with six leaves). Different letters indicate significant difference by Fisher's least significant difference test $(P<0.05)$.

vesiculogen-containing, and slightly more rounded. Sporangia from other 5 empty-vector transformants also displayed similar characteristics. In contrast, the mean number of sporangia for PpMID1_7 and PpMID1_46 was $446 \pm 31$ and $600 \pm 93$ per 2-mm-square agar block (Table 1), respectively, significantly less than the wild type and EV. Moreover, all these sporangia were non-papillate and round-shaped, from 10 to $25 \mu \mathrm{m}$ in diameter, and lacked vesiculogen (Figure 5). When cold-shocked to induce zoospore formation, sporangia of the wild type and EV released a mean of $755 \pm 79$ and $873 \pm 60$ zoospores per $10 \mu \mathrm{L}$ induction solution, respectively. In contrast, sporangia of PpMID1_7 were unable to release any zoospores. Instead, they tended to germinate directly, with 3 to 15 germ tubes sprouting from the round-shaped sporangia (Figure 5). Most sporangia produced by $P$ PMID1_46 germinated directly, producing 1 to 3 germ tubes, and some produced a very small number of zoospores (Table 1), which were usually two to three times larger than normal ones. Thus, PpMID1 silencing interfered with the process of not only sporangium formation but also zoospore production.

\section{PpMID1-silenced Transformants Show Defects in Cytoplasmic Cleavage of Sporangia}

To determine whether the failure to release zoospores results from a defect in zoospore differentiation or zoospore release, we stained sporangia with DAPI and the lipophilic dye FM 4-64 after cold shock. DAPI staining revealed the presence of multiple nuclei in sporangia of the wild type and EV, which distributed evenly in the sporangial cytoplasm (Figure 6). Moreover, staining with FM 4-64 for plasma membrane demonstrated the occurrence of sporangial cytoplasmic cleavage in response to cold shock (Figure 6). The silenced sporangia showed multiple nuclei after DAPI staining but no sign of cytoplasmic cleavage on cold shock (Figure 6). Hence, PpMID1 silencing impaired the process of cytoplasmic cleavage. As a result, silenced sporangia could not produce zoospores.

\section{Addition of $\mathrm{CaCl}_{2}$ or $\mathrm{MgCl}_{2}$ Recovers the Morphology of Sporangia in PpMID1-silenced Transformants and Reclaims Their Ability to Produce \\ Zoospores}

PpMID1 is predicted to encode a subunit of the high-affinity $\mathrm{Ca}^{2+}$ channel. PpMID1 silencing likely disturbs the influx of calcium ions to impair the process of sporangium formation and cytoplasmic cleavage. To test this hypothesis, mycelia blocks of silenced transformants were incubated in $1 \% \mathrm{~V} 8$ juice medium amended with $50 \mathrm{mM} \mathrm{CaCl} 2$ under light to induce sporangium formation. As controls, similar experiments were performed by replacing $\mathrm{CaCl}_{2}$ with $50 \mathrm{mM} \mathrm{MgCl}_{2}$ (an alternative cation) or $150 \mathrm{mM}$ mannitol (a non-ionic osmolite). Moreover, to avoid the effect caused by a difference in osmolarity, each medium was adjusted to the same osmotic pressure according to the van't Hoff law. The wild type and EV produced papillate, ovoid sporangia with $\mathrm{CaCl}_{2}, \mathrm{MgCl}_{2}$, or mannitol in the medium (Figures 7A-H). Remarkably, the addition of $\mathrm{CaCl}_{2}$ or $\mathrm{MgCl}_{2}$ reversed the morphology of some sporangia produced by PpMID1_7 and PpMID1_46 (Table 2). These sporangia became papillate, ovoid, and bore vesiculogen, similar to sporangia produced by the wild type (Figures 7J,K,N,O). However, the papillae of sporangia produced by PpMID1_7 were slightly enlarged and irregular-shaped (Figures 7J,K). In contrast, with mannitol amendment, the silenced sporangia remained unaltered (Table 2 and Figures 7L,P), similar to those produced in the absence of any amendment (Figures 7I,M). Further staining with FM 4-64 revealed rescued cytoplasmic cleavage in silenced sporangia with the addition of $\mathrm{CaCl}_{2}$ or $\mathrm{MgCl}_{2}$ (Figures 7R,S,V,W), but not mannitol (Figures 7T,X) and the control without any amendment (Figures 7Q,U).

\section{PpMID1-silencing Has No Effect on Oospore Formation}

$M I D 1$ plays a key role in the mating process of S. cerevisiae (Iida et al., 1994). To determine whether PPMID1 is also involved in the sexual reproduction of $P$. parasitica, we performed mating 


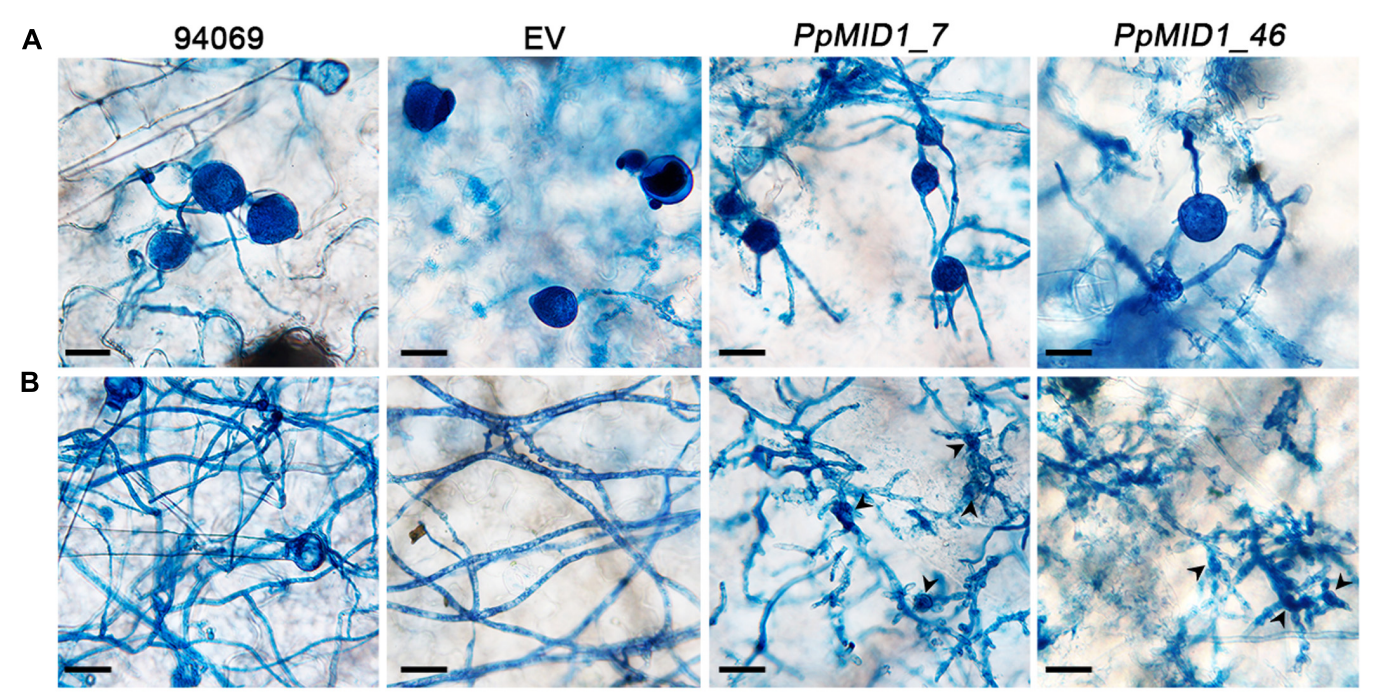

FIGURE 10 | PpMID1-silenced transformants generate highly branched mycelia and aberrant sporangia. Mycelia disks (5 mm id.) of PpMID1_7, PpMID1_46 or wild-type Phytophthora parasitica (94069) were transferred to the center of leaves from 5-week-old Nicotiana benthamiana. After incubation in a moisture box at $25^{\circ} \mathrm{C}$ for $72 \mathrm{~h}$, inoculated leaves were stained with trypan blue and photographed under a microscope. (A) Silenced transformants produced sporangia that germinated directly. Bar $=25 \mu \mathrm{m}$. (B) Silenced transformants tended to form deformed and highly branched mycelia. Arrowheads, bulged mycelia of the silenced transformants. Bar $=25 \mu \mathrm{m}$.

experiments and counted the number of resultant oospores. The wild-type strain (94069) used for generating the silenced strains has the A2 mating type. On mating PpMID1_7 or PpMID_46 with a $P$. parasitica isolate of the A1 mating type (isolate number 991), the formation of oospores was similar both in number and morphology to those formed on mating of the wild type (94069) or EV with the A1 strain (Figure 8). Therefore, PpMID1 silencing had no effect on the mating and oospore formation of P. parasitica.

\section{PpMID1-silenced Transformants Show Reduced Virulence and Abnormal Features in Infected Plants}

To determine whether downregulation of PPMID1 affects the virulence of $P$. parasitica, we inoculated detached leaves of 5-week-old $N$. benthamiana with mycelia disks of the PpMID1-silenced transformants. When inoculated with the wild-type $P$. parasitica, the leaves showed water-soaking symptom that covered approximately half of the leaf area by 48 hpi. The lesion then enlarged to encompass almost the whole leaf by 72 hpi (Figure 9A). In contrast, in the leaves inoculated with either silenced strain, the disease symptom was alleviated (Figure 9A), with a significant difference between infected areas caused by the wild type and silenced transformants (Figure 9B), although the EV showed reduced virulence as well. To discover why PpMID1 silencing reduced the virulence of $P$. parasitica, we stained infected $N$. benthamiana leaves with trypan blue. Microscopy examination revealed the presence of abundant sporangia on $N$. benthamiana leaves infected by the wild type at 72 hpi (Figure 10A), accompanied by the presence of some zoospores nearby. In contrast, sporangia were barely seen on leaves infected with PpMID1_7 or PpMID1_46. Of the 10 leaves examined, only one contained sporangia that germinated directly. Moreover, branched hyphae often bulged in the middle or ends (Figure 10B, arrowheads). Thus, PpMID1 silencing affects the virulence of $P$. parasitica, likely caused by a failure in normal hyphal branching and sporangium formation.

\section{DISCUSSION}

Calcium signaling is involved in the regulation of asexual reproduction in $P$. parasitica, which encompasses sporangium formation and zoospore differentiation (von Broembsen and Deacon, 1997; Warburton and Deacon, 1998). Despite previous efforts to elucidate the underlying mechanism, knowledge about the relevant signaling components has been limited, especially those involved in calcium transportation. Here, we demonstrate that PpMID1 is essential for the asexual reproduction of this pathogen.

PpMID1 is a homolog of MID1 that together with $\mathrm{CCH} 1$ forms the only high-affinity calcium channel in the plasma membrane of fungal cells (Liu et al., 2006). S. cerevisiae MID1 contains an ORF of 548 aa and alone is capable of forming a calciumpermeable, cation-selective stretch-activated channel in Chinese hamster ovary cells (Kanzaki et al., 1999). In contrast, MID1 of C. neoformans with 558 aa does not show any independent channel activity under calcium depletion conditions (Hong et al., 2010). The predicted amino acid sequence and size of PpMID1 are similar to its homologs from other Oomycetes. However, these proteins are conserved only to the C-terminal regions of fungal MID1, especially the highly conserved cysteine residues (Figure 1). In S. cerevisiae, these cysteine residues are essential 
for the function of MID1 (Maruoka et al., 2002). As well, they are likely involved in the formation of MID1 oligomeric structures that are associated with CCH1 (Locke et al., 2000; Hong et al., 2010, 2013). In this regard it is interesting to note that, despite the identification of MID1 homologs in P. parasitica and other oomycetes, no $\mathrm{CCH} 1$ homolog is found in oomycetes according to the information obtained from FungiDB (Stajich et al., 2012). Further work is required to determine whether PpMID1 shows independent channel activity similar to yeast MID1.

Silencing of PpMID1 had no effect on the growth rate of $P$. parasitica (Table 1). However, it did cause defective hyphal branching and aerial hypha formation, likely due to disturbed calcium homeostasis in the mycelia of $P$. parasitica. Of note, intracellular calcium concentrations regulate hyphal extension, orientation and branching in various fungi. Low calcium level caused enhanced hyphal branching in Fusarium graminearum and N. crassa (Dicker and Turian, 1990; Robson et al., 1991a,b), and chelation of extracellular calcium or deletion of MID1 and CCH1 disturbed thigmotropism and severely reduced curve formation in C. albicans (Brand et al., 2007, 2009). A recent study demonstrated highly branched hyphae of an A. nidulans MID1 mutant (midA) showing at the hyphal apex an aberrant distribution pattern of the Spitzenkörper (Wang et al., 2012). Nonetheless, not much is known about the regulation of hyphal branching in oomycetes. Additional studies are needed to determine the mechanism that underlies regulation of hyphal branching by calcium in these organisms.

In response to cold shock, sporangia of Phytophthora spp. undergo cytoplasmic cleavage, followed by the formation and release of zoospores. Our PpMID1-silenced transformants produced fewer sporangia with abnormal morphology: roundshaped and lacking papilla. Moreover, sporangia tended to germinate directly or, owing to failure of cytoplasmic cleavage as revealed by FM 4-64 staining, formed none or only a very small number of zoospores. In P. cinnamomi, a transient increase in calcium concentration in the sporangial cytoplasm was required for the initiation of cytoplasmic cleavage in sporangia (Jackson and Hardham, 1996). As well, treatments to interfere with calcium homeostasis disturbed sporangia germination and zoospore release in P. infestans (Hill et al., 1998; Judelson and Roberts, 2002). The failure of our PpMID1silenced transformants to produce normal sporangia and to release zoospores was likely caused by reduced expression of PpMID1 and thereby interference in calcium influx. Studies of various fungal species indicated that calcium supplementation could rescue the aberrant phenotypes of MID1 mutants in various fungi (Iida et al., 1994; Cavinder et al., 2011; Wang et al., 2012; Harren and Tudzynski, 2013). In our study, the addition of $\mathrm{CaCl}_{2}$ substantially reversed the morphology of silenced sporangia and recovered cytoplasmic cleavage in response to cold shock, which supports an essential role of PpMID1 in calcium signaling. Not all sporangia recovering cleavage was likely due to differential silencing effects of PpMID1. Intriguingly, the addition of $\mathrm{MgCl}_{2}$ could also reverse the morphology and recover cytoplasmic cleavage in the silenced sporangia. In concert with our results, magnesium could substitute for calcium to rescue the suppression of sporangial cleavage and zoospore release imposed by calcium chelators in P. infestans (Hill et al., 1998). Magnesium is one of the most abundant divalent cations in cells, with its cellular content tightly regulated. RNA profile analysis performed in S. cerevisiae demonstrated a close and complicated relationship between magnesium and calcium homeostasis (Wiesenberger et al., 2007). Moreover, magnesium may bind calmodulin and other EF-hand proteins, thereby modulating their calcium binding activity (Grabarek, 2011). Nonetheless, these studies pointed to the possibility that an excess of magnesium may attenuate calcium signaling. Further study is required to define the possible alternative pathway(s) adopted by PpMID1-silenced transformants to restore the aberrant phenotypes with the addition of $\mathrm{MgCl}_{2}$.

In S. cerevisiae, MID1 mutants die on receiving the opposite mating hormone, which indicates the important role of the molecule in the mating process of yeast (Iida et al., 1994). In Fusarium graminearum (sexual-stage Gibberella zeae), the agent causing head blight in wheat and barley, deletion of MID1 resulted in the production of predominately abnormal ascospores and a sharp reduction in ascospore discharge activity (Cavinder et al., 2011). However, MID1 mutants of $N$. crassa could mate and produce viable ascospores (Lew et al., 2008). Similarly, silencing PpMID1 had no effect on the mating process of $P$. parasitica. Therefore, the role of MID1 in sexual reproduction may vary depending on the organism.

In summary, reduced expression of PpMID1 can cause severe defects in hyphal branching, sporangium formation, and cytoplasmic cleavage in sporangia of $P$. parasitica, which supports an important role of PPMID1 in asexual reproduction in this species. The inability to produce normal hyphae and sporangia can also reduce the virulence of the silenced transformants. Further effort is required to elucidate how PPMID1 is involved in the modulation of calcium homeostasis in this pathogen.

\section{AUTHOR CONTRIBUTIONS}

Conceived and designed the experiments: FYH, MWL, and RFL. Contributed to reagents/materials/analysis tools: RFL. Performed the experiments: FYH and MWL. Analyzed the data: FYH, MWL, and RFL. Wrote the manuscript: FYH, MWL, and RFL.

\section{FUNDING}

The research reported in this publication was supported by funding from the Ministry of Science and Technology, Taiwan.

\section{ACKNOWLEDGMENTS}

We are grateful to Dr. P. J. Ann (Taiwan Agricultural Research Institute) for providing P. parasitica isolates, to Drs. Y. C. 
Wang (Department of Plant Pathology, Nanjing Agricultural University) and H. Judelson (Department of Plant Pathology and Microbiology, University of California, Riverside) for providing the plasmid vectors, and to Dr. Y. C. Wang for technical assistance.

\section{REFERENCES}

Baldauf, S. L., Roger, A. J., Wenk-Siefert, I., and Doolittle, W. F. (2000). A kingdomlevel phylogeny of eukaryotes based on combined protein data. Science 290, 972-977. doi: 10.1126/science.290.5493.972

Bonilla, M., Nastase, K. K., and Cunningham, K. W. (2002). Essential role of calcineurin in response to endoplasmic reticulum stress. EMBO J. 21, 2343-2353. doi: 10.1093/emboj/21.10.2343

Brand, A., Lee, K., Veses, V., and Gow, N. A. R. (2009). Calcium homeostasis is required for contact-dependent helical and sinusoidal tip growth in Candida albicans hyphae. Mol. Microbiol. 71, 1155-1164. doi: 10.1111/j.1365-2958.2008. 06592.x

Brand, A., Shanks, S., Duncan, V. M., Yang, M., Mackenzie, K., and Gow, N. A. R. (2007). Hyphal orientation of Candida albicans is regulated by a calciumdependent mechanism. Curr. Biol. 17, 347-352. doi: 10.1016/j.cub.2006. 12.043

Cavinder, B., Hamam, A., Lew, R. R., and Trail, F. (2011). Mid1, a mechanosensitive calcium ion channel, affects growth, development, and ascospore discharge in the filamentous fungus Gibberella zeae. Eukaryot. Cell 10, 832-841. doi: 10.1128/EC.00235-10

Christen, J., and Hohl, H. R. (1972). Growth and ultrastructural differentiation of sporangia in Phytophthora palmivora. Can. J. Microbiol. 18, 1959-1964. doi: $10.1139 / \mathrm{m} 72-303$

Dicker, J. W., and Turian, G. (1990). Calcium deficiencies and apical hyperbranching in wild-type and the "frost" and "spray" morphological mutants of Neurospora crassa. J. Gen. Microbiol. 136, 1413-1420. doi: 10.1099/ 00221287-136-7-1413

Ding, X., Yu, Q., Xu, N., Wang, Y., Cheng, X., Qian, K., et al. (2013). Ecm7, a regulator of HACS, functions in calcium homeostasis maintenance, oxidative stress response and hyphal development in Candida albicans. Fungal Genet. Biol. 57, 23-32. doi: 10.1016/j.fgb.2013.05.010

Erwin, D. C., and Ribeiro, O. K. (1996). Phytophthora Diseases Worldwide. St. Paul, MN: APS Press.

Fischer, M., Schnell, N., Chattaway, J., Davies, P., Dixon, G., and Sanders, D. (1997). The Saccharomyces cerevisiae CCH1 gene is involved in calcium influx and mating. FEBS Lett. 419, 259-262. doi: 10.1016/S0014-5793(97)01466-X

Grabarek, Z. (2011). Insights into modulation of calcium signaling by magnesium in calmodulin, troponin C and related EF-hand proteins. Biochim. Biophys. Acta 1813, 913-921. doi: 10.1016/j.bbamcr.2011.01.017

Hardham, A. R. (2001). The cell biology behind Phytophthora pathogenicity. Austral. Plant Pathol. 30, 91-98. doi: 10.1071/AP01006

Harren, K., and Tudzynski, B. (2013). Cch1 and Mid1 are functionally required for vegetative growth under low-calcium conditions in the phytopathogenic ascomycete Botrytis cinerea. Eukaryot. Cell 12, 712-724. doi: 10.1128/EC. 00338-12

Hemmes, D. E. (1983). “Cytology of Phytophthora”, in Phytophthora: Its Biology, Taxonomy, Ecology, and Pathology, eds D. C. Erwin, S. Bartnicki-Garcia, and P. H. Tsao (St. Paul, MN: APS Press), 9-40.

Hill, A. E., Grayson, D. E., and Deacon, J. W. (1998). Suppressed germination and early death of Phytophthora infestans sporangia caused by pectin, inorganic phosphate, ion chelators and calcium-modulating treatments. Eur. J. Plant Pathol. 104, 367-376. doi: 10.1023/A:1008660129390

Hong, M. P., Vu, K., Bautos, J. M., and Gelli, A. (2010). Cch1 restores intracellular $\mathrm{Ca} 2+$ in fungal cells during endoplasmic reticulum stress. J. Biol. Chem. 285, 10951-10958. doi: 10.1074/jbc.M109.056218

Hong, M. P., Vu, K., Bautos, J. M., Tham, R., Jamklang, M., Uhrig, J. P., et al. (2013). Activity of the calcium channel pore $\mathrm{Cch} 1$ is dependent on a modulatory region of the subunit Mid1 in Cryptococcus neoformans. Eukaryot. Cell 12, 142-150. doi: 10.1128/EC.00130-12

\section{SUPPLEMENTARY MATERIAL}

The Supplementary Material for this article can be found online at: http://journal.frontiersin.org/article/10.3389/fmicb. 2017.00610/full\#supplementary-material

Hua, C., Meijer, H. J., de Keijzer, J., Zhao, W., Wang, Y., and Govers, F. (2013). GK4, a G-protein-coupled receptor with a phosphatidylinositol phosphate kinase domain in Phytophthora infestans, is involved in sporangia development and virulence. Mol. Microbiol. 88, 352-370. doi: 10.1111/mmi.12190

Hyde, G. J., Lancelle, S., Hepler, P. K., and Hardham, A. R. (1991). Freeze substitution reveals a new model for sporangial cleavage in Phytophthora, a result with implications for cytokinesis in other eukaryotes. J. Cell Sci. 100, 735-749.

Iida, H., Nakamura, H., Ono, T., Okumura, M. S., and Anraku, Y. (1994). MID1, a novel Saccharomyces cerevisiae gene encoding a plasma membrane protein, is required for $\mathrm{Ca}^{2+}$ influx and mating. Mol. Cell. Biol. 14, 8259-8271. doi: $10.1128 / \mathrm{mcb} .14 .12 .8259$

Jackson, S. L., and Hardham, A. R. (1996). A transient rise in cytoplasmic free calcium is required to induce cytokinesis in zoosporangia of Phytophthora cinnamomi. Eur. J. Cell Biol. 69, 180-188.

Judelson, H. S., Ah-Fong, A. M., Aux, G., Avrova, A. O., Bruce, C., Cakir, C., et al. (2008). Gene expression profiling during asexual development of the late blight pathogen Phytophthora infestans reveals a highly dynamic transcriptome. Mol. Plant Microbe Interact. 21, 433-447. doi: 10.1094/MPMI-21-4-0433

Judelson, H. S., and Michelmore, R. W. (1991). Transient expression of genes in the oomycete Phytophthora infestans using Bremia lactucae regulatory sequences. Curr. Genet. 19, 453-459. doi: 10.1007/bf00312736

Judelson, H. S., Narayan, R. D., Ah-Fong, A. M., and Kim, K. S. (2009). Gene expression changes during asexual sporulation by the late blight agent Phytophthora infestans occur in discrete temporal stages. Mol. Genet. Genomics 281, 193-206. doi: 10.1007/s00438-008-0407-5

Judelson, H. S., and Roberts, S. (2002). Novel protein kinase induced during sporangial cleavage in the Oomycete Phytophthora infestans. Eukaryot. Cell 1, 687-695. doi: 10.1128/EC.1.5.687-695.2002

Judelson, H. S., and Tani, S. (2007). Transgene-induced silencing of the zoosporogenesis-specific NIFC gene cluster of Phytophthora infestans involves chromatin alterations. Eukaryot. Cell 6, 1200-1209. doi: 10.1128/EC.00311-06

Judelson, H. S., Tyler, B. M., and Michelmore, R. W. (1991). Transformation of the oomycete pathogen, Phytophthora infestans. Mol. Plant Microbe Interact. 4, 602-607. doi: 10.1094/mpmi-4-602

Kanzaki, M., Nagasawa, M., Kojima, I., Sato, C., Naruse, K., Sokabe, M., et al. (1999). Molecular identification of a eukaryotic, stretch-activated nonselective cation channel. Science 285, 882-886. doi: 10.1126/science.285.5429.882

Kim, K. S., and Judelson, H. S. (2003). Sporangium-specific gene expression in the oomycete phytopathogen Phytophthora infestans. Eukaryot. Cell 2, 1376-1385. doi: 10.1128/EC.2.6.1376-1385.2003

Latijnhouwers, M., and Govers, F. (2003). A Phytophthora infestans G-protein beta subunit is involved in sporangium formation. Eukaryot. Cell 2, 971-977. doi: 10.1128/EC.2.5.971-977.2003

Lew, R. R., Abbas, Z., Anderca, M. I., and Free, S. J. (2008). Phenotype of a mechanosensitive channel mutant, mid-1, in a filamentous fungus, Neurospora crassa. Eukaryot. Cell 7, 647-655. doi: 10.1128/EC.00411-07

Liu, M., Du, P., Heinrich, G., Cox, G. M., and Gelli, A. (2006). Cch1 mediates calcium entry in Cryptococcus neoformans and is essential in low-calcium environments. Eukaryot. Cell 5, 1788-1796. doi: 10.1128/EC.00158-06

Locke, E. G., Bonilla, M., Liang, L., Takita, Y., and Cunningham, K. W. (2000). A homolog of voltage-gated $\mathrm{Ca}^{2+}$ channels stimulated by depletion of secretory $\mathrm{Ca}^{2+}$ in yeast. Mol. Cell. Biol. 20, 6686-6694. doi: 10.1128/mcb.20.18.66866694.2000

Maltese, C. E., Conigliaro, G., and Shaw, D. S. (1995). The development of sporangia of Phytophthora infestans. Mycol. Res. 99, 1175-1181. doi: 10.1016/ s0953-7562(09)80273-6

Martin, D. C., Kim, H., Mackin, N. A., Maldonado-Báez, L., Evangelista, C. C. Jr., Beaudry, V. G., et al. (2011). New regulators of a high affinity $\mathrm{Ca}^{2+}$ influx 
system revealed through a genome-wide screen in yeast. J. Biol. Chem. 286, 10744-10754. doi: 10.1074/jbc.M110.177451

Maruoka, T., Nagasoe, Y., Inoue, S., Mori, Y., Goto, J., Ikeda, M., et al. (2002). Essential hydrophilic carboxyl-terminal regions including cysteine residues of the yeast stretch-activated calcium-permeable channel Mid1. J. Biol. Chem. 277, 11645-11652. doi: 10.1074/jbc.m111603200

McLeod, A., Fry, B. A., Zuluaga, A. P., Myers, K. L., and Fry, W. E. (2008). Toward improvements of oomycete transformation protocols. J. Eukaryot. Microbiol. 55, 103-109. doi: 10.1111/j.1550-7408.2008.00304.x

Muller, E. M., Locke, E. G., and Cunningham, K. W. (2001). Differential regulation of two $\mathrm{Ca}^{2+}$ influx systems by pheromone signaling in Saccharomyces cerevisiae. Genetics 159, 1527-1538.

Muller, E. M., Mackin, N. A., Erdman, S. E., and Cunningham, K. W. (2003). Figlp facilitates $\mathrm{Ca}^{2+}$ influx and cell fusion during mating of Saccharomyces cerevisiae. J. Biol. Chem. 278, 38461-38469. doi: 10.1074/jbc.M304089200

Ribeiro, O. K. (1983). "Physiology of asexual sporulation and spore germination," in Phytophthora: Its Biology, Taxonomy, Ecology, and Pathology, eds D. C. Erwin, S. Bartnicki-Garcia, and P. H. Tsao (St. Paul, MN: APS Press), 55-70.

Robson, G. D., Wiebe, M. G., and Trinci, A. P. J. (1991a). Involvement of $\mathrm{Ca}^{2+}$ in the regulation of hyphal extension and branching in Fusarium graminearum A 3/5. Exp. Mycol. 15, 263-272. doi: 10.1016/0147-5975(91)90028-C

Robson, G. D., Wiebe, M. G., and Trinci, A. P. J. (1991b). Low calcium concentrations induce increased branching in Fusarium graminearum. Microbiol. Res. 95, 561-565. doi: 10.1016/s0953-7562(09)80069-5

Stajich, J. E., Harris, T., Brunk, B. P., Brestelli, J., Fischer, S., Harb, O. S., et al. (2012). FungiDB: an integrated functional genomics database for fungi. Nucleic Acids Res. 40, D675-D681. doi: 10.1093/nar/gkr918

Tani, S., Yatzkan, E., and Judelson, H. S. (2004). Multiple pathways regulate the induction of genes during zoosporogenesis in Phytophthora infestans. Mol. Plant Microbe Interact. 17, 330-337. doi: 10.1094/MPMI.2004.17.3.330

Thewes, S. (2014). Calcineurin-Crz1 signaling in lower eukaryotes. Eukaryot. Cell 13, 694-705. doi: 10.1128/EC.00038- 14

von Broembsen, S. L., and Deacon, J. W. (1997). Calcium interference with zoospore biology and infectivity of Phytophthora parasitica in nutrient irrigation solutions. Phytopathology 87, 522-528. doi: 10.1094/PHYTO.1997.87. 5.522

Wang, S., Cao, J. L., Liu, X., Hu, H. Q., Shi, J., Zhang, S. Z., et al. (2012). Putative calcium channels CchA and MidA play the important roles in conidiation, hyphal polarity and cell wall components in Aspergillus nidulans. PLoS ONE 7:e46564. doi: 10.1371/journal.pone.0046564

Warburton, A. J., and Deacon, J. W. (1998). Transmembrane $\mathrm{Ca}^{2+}$ fluxes associated with zoospore encystment and cyst germination by the phytopathogen Phytophthora parasitica. Fungal Genet. Biol. 25, 54-62. doi: 10.1006/fgbi.1998.1086

Wiesenberger, G., Steinleitner, K., Malli, R., Graier, W. F., Vormann, J., Schweyen, R. J., et al. (2007). $\mathrm{Mg}^{2+}$ deprivation elicits rapid $\mathrm{Ca}^{2+}$ uptake and activates $\mathrm{Ca}^{2+} /$ calcineurin signaling in Saccharomyces cerevisiae. Eukaryot Cell. 6, 592-599. doi: 10.1128/EC.00382-06

Wilson, U. E., and Coffey, M. D. (1980). Cytological evaluation of general resistance to Phytophthora infestans in potato foliage. Ann. Bot. 45, 81-90.

Yan, H. Z., and Liou, R. F. (2006). Selection of internal control genes for realtime quantitative RT-PCR assays in the oomycete plant pathogen Phytophthora parasitica. Fungal Genet. Biol. 43, 430-438. doi: 10.1016/j.fgb.2006.01.010

Zhang, M., Lu, J., Tao, K., Ye, W., Li, A., Liu, X., et al. (2012). A Myb transcription factor of Phytophthora sojae, regulated by MAP kinase PSSAK1, is required for zoospore development. PLOS ONE 7:e40246. doi: 10.1371/journal.pone. 0040246

Conflict of Interest Statement: The authors declare that the research was conducted in the absence of any commercial or financial relationships that could be construed as a potential conflict of interest.

Copyright (c) $2017 \mathrm{Hwu}$, Lai and Liou. This is an open-access article distributed under the terms of the Creative Commons Attribution License (CC BY). The use, distribution or reproduction in other forums is permitted, provided the original author(s) or licensor are credited and that the original publication in this journal is cited, in accordance with accepted academic practice. No use, distribution or reproduction is permitted which does not comply with these terms. 